\title{
Leitthema
}

Bundesgesundheitsbl 2015 · 58:699-704

DOI 10.1007/s00103-015-2160-3

Online publiziert: 12. Mai 2015

c) Springer-Verlag Berlin Heidelberg 2015
Martin Richter · Christian Herzog

Zentrum für Biologische Gefahren und Spezielle Pathogene, Informationsstelle des Bundes für

Biologische Gefahren und Spezielle Pathogene, Robert Koch-Institut, Berlin, Deutschland

\section{Allgemeines Vorgehen bei Verdacht auf einen Anschlag mit hochkontagiösen, hochpathogenen Agenzien}

\section{Einleitung}

Bioterrorismus nach Beeching et al. und Jansen et al. bezeichnet das absichtliche Freisetzen von biologischen Agenzien im Rahmen eines terroristischen Anschlages mit dem Ziel, der Zivilbevölkerung oder zivilen Strukturen Schaden zuzufügen, um politische, ideologische, vergeltende oder religiöse Ziele zu verfolgen bzw. erpresserisch Forderungen durchzusetzen $[1,2]$. Zurückblickend auf die Anzahl bisher stattgefundener terroristischer Ereignisse lässt sich schließen, dass bei terroristischen Gruppierungen bislang äußert selten die Absicht bestand, biologische Agenzien im Rahmen von Anschlagsvorhaben einzusetzen. Dies kann zum einen in der fehlenden Expertise zur Präparation hochansteckenden Materials in ausreichender Konzentration und Menge, aber zum anderen auch in den limitierten Möglichkeiten zur Beschaffung relevanter biologischer Agenzien begründet sein. Des Weiteren spielt möglicherweise auch die unmittelbar angestrebte Wirkung des Anschlages eine Rolle. Anschläge mit biologischen Agenzien sind zum Beispiel im Vergleich zu Sprengstoffanschlägen aufgrund der Inkubationszeit bis zum Ausbruch einer Erkrankung nicht sofort offensichtlich und eignen sich somit nicht als akut präsentes Schlüsselereignis. Natürliche Ausbruchsgeschehen, wie die Ebolafieber-Epidemie in Westafrika $[3,4]$ oder die Pestausbrüche in Madagaskar [5], aber auch Seuchengeschehen in der westlichen Welt, wie die Influenzapandemie 2009/2010 [6], zeigen jedoch eindrucksvoll, welch hohes Scha- densausmaß mit möglicherweise langwierigen Folgen für die Zivilbevölkerung und die Gesundheitsstrukturen eines Landes ein Anschlag mit hochkontagiösen bzw. hochpathogenen Agenzien bergen kann. Das Pflanzengift Rizin, Humanpockenviren, Ebolaviren und Pestbakterien stellen einige Vertreter dar, die von Fachkreisen als bioterroristisch relevante Agenzien eingeschätzt werden $[7,8]$. Die bislang folgenreichste absichtliche Ausbringung eines biologischen Agens in der Neuzeit liegt mit den Milzbrandbriefen, die in den USA im Nachgang zu den Anschlägen am 11. September 2001 versandt wurden, über 10 Jahre zurück. Die jüngsten Fälle, die weltweit für Aufmerksamkeit sorgten, sind Briefsendungen mit dem Pflanzengift Rizin aus dem Jahr 2013 an den Präsidenten der USA, einen Senator und den damaligen Bürgermeister von New York.

Auch wenn es in Deutschland noch nie zu einem dokumentierten Bioterrorismusereignis gekommen ist, dienen vor allem die Anschläge aus dem Jahr 2001 immer wieder und bis heute als Vorlage für Nachahmungstäter, die entsprechend präparierte Pulverbriefe, jedoch ohne Beifügen hochpathogener Agenzien, an bestimmte Personen, Personengruppen oder Institutionen versenden. Vor allem aus diesem Grund muss dem Vorgehen bei vermuteter absichtlicher Ausbringung von hochpathogenen Agenzien eine umfassende Ernsthaftigkeitsprüfung vorausgehen, die sich u. a. auf aktuelle Gefährdungsbewertungen von Sicherheitsbehörden stützen kann. Letztlich bestimmt die ereignisbezogene Ernsthaftigkeitsprüfung alle Folgemaßnahmen als Reaktion auf ein solches Ereignis, die dann in vollständiger Konsequenz inklusive seuchenhygienischer Maßnahmen durchgeführt werden müssen.

Die absichtliche Ausbringung hochpathogener Agenzien kann auf verschiedenen Wegen erfolgen [9]. Der Verdacht auf eine Beimischung dieser Agenzien wird in der Realität meistens bei Postsendungen geäußert, die undefinierbare Pulver enthalten. Daher wird hier besonders auf das Vorgehen beim Auffinden von Pulversendungen eingegangen.

\section{Erkennen, Ernsthaf- tigkeitsprüfung und Gefährdungsbewertung}

Bei der Gefährdungsbewertung wird zwischen konkreter Gefahr und abstrakter Gefahr unterschieden. Eine konkrete Gefahr ergibt sich aus unmittelbaren Zuständen, die bei weitergehendem Ablauf mit entsprechend hoher Wahrscheinlichkeit zum schädigenden Ereignis führen. Bei der abstrakten Gefahr handelt es sich um ein nach den Erkenntnissen fachkundiger Stellen vorliegendes Gefahrenpotenzial (gedachter Sachverhalt), das zu einer konkreten Gefahr führen kann. Dies ist abhängig von Informationen und Hinweisen, die das theoretische Eintreten eines schädigenden Ereignisses plausibilisieren [10].

Aus einer abstrakten Gefahr wird somit bei Vorliegen eines konkreten Sachverhaltes (z. B. Ort, Zeit und Art des Schadenseintritts sind erkennbar) eine konkrete Gefahr. Theoretisch wird vor einem Schadenseintritt aus einer abstrak- 


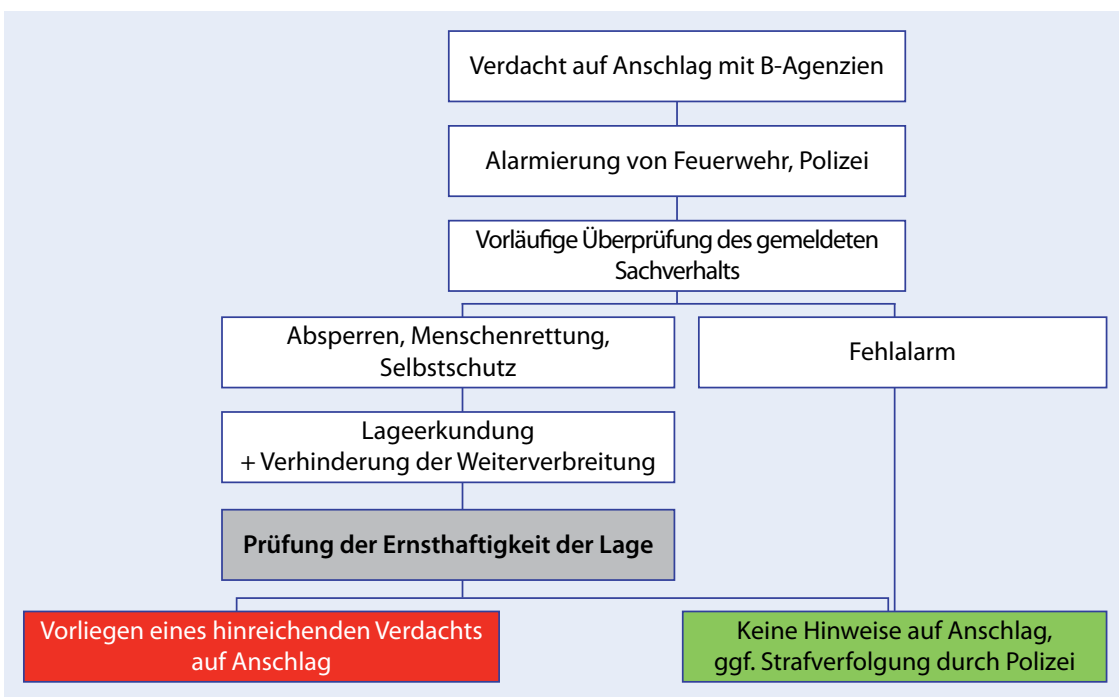

Abb. $1 \Delta$ Vorgehen bei vermutlicher Ausbringung eines B-Agens; erste Maßnahmen und Ernsthaftigkeitsprüfung

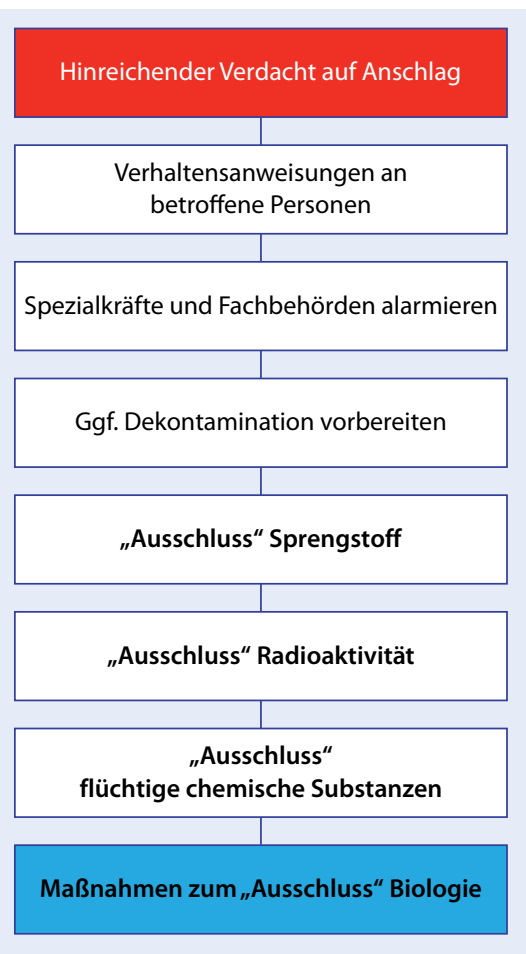

Abb. $2 \Delta$ Vorgehen bei vermutlicher Ausbringung eines B-Agens; Vorgehen bei hinreichendem Verdacht auf einen Anschlag

ten Gefahr immer erst eine konkrete Gefahr. Außerhalb einer idealen Betrachtung kann die abstrakte Gefahr direkt zu einem Schadenseintritt führen, falls Hinweise auf den konkreten einzelnen Sachverhalt vor Schadenseintritt den fachkundigen Stellen nicht vorliegen bzw. nicht erkannt werden.
Aufgrund der zahlreichen harmlosen Pulversendungen der vergangenen Jahre lässt sich bei unveränderter Gefährdungslage mit aller Vorsicht vermuten, dass auch bei zukünftigen Pulverfunden eine absichtliche Beimischung von hochpathogenen Agenzien wenig wahrscheinlich sein dürfte. Daher sollte bei Pulverfunden zunächst grundsätzlich die Ernsthaftigkeit der Lage überprüft werden (z. B. liegt eine veränderte abstrakte Gefahr bzw. eine konkrete Gefahr eines bioterroristischen Anschlags vor), um einerseits keine unnötigen Maßnahmen zu ergreifen, aber andererseits auch keine reale Gefahr zu übersehen. Die Prüfung der Ernsthaftigkeit der Lage erfolgt in der Regel durch die zuständigen Polizeibehörden, die bei Bedarf Informationen von ihren Landes- und Bundesbehörden einbeziehen können. Das Ergebnis dieser Überprüfung determiniert dann das weitere Vorgehen. Darunter auch, ob überhaupt ein Verdacht der Beimischung nicht konventioneller Stoffe (chemische, radioaktive, biologische) Bestand haben kann oder ob eine stoffliche Untersuchung notwendig wird (• Abb. 1).

Das Vorhandensein berechtigter Hinweise auf einen Anschlag bzw. eine absichtliche Ausbringung nicht konventioneller Stoffe ist demnach ausschlaggebend. Folgende Fragestellungen können bei der Bewertung der Ernsthaftigkeit der Lage hilfreich sein:
- Liegt eine erhöhte abstrakte Gefährdungslage für terroristische Anschläge mit nicht konventionellen Stoffen vor?

- Sind konkrete Hinweise auf Bedrohung bzw. einen möglichen Anschlag vorhanden?

- Besteht ein Bezug zu sicherheitsgefährdeten Personen, Veranstaltungen oder kritischen Infrastrukturen?

- Weisen Geräte/technische Konstruktionen auf absichtliche Ausbringung von Agenzien hin?

\section{Besonderheit bei Ausbringung hochpathogener Agenzien}

Weil eine Infektion nach Exposition von Personen mit einem hochpathogenen Agens nicht unmittelbar zu sichtbaren Beeinträchtigungen der Betroffenen führt, ist es generell möglich, dass eine absichtliche Ausbringung derartiger Agenzien erst dann auffällig wird, wenn die entsprechenden Personen nach Beginn von Symptomen z. B. ärztliche Hilfe aufsuchen. Abhängig von der Inkubationszeit kann dann das eigentliche Ereignis der Infektion einige Stunden bis mehrere Tage zurückliegen, also völlig unbemerkt stattgefunden haben. Auffälligkeiten werden dann erst erfasst, wenn z. B. nach unbeobachteter Ausbringung eines hochpathogenen Agens ein Cluster ungewöhnlicher Erkrankungen auftritt und dieser auch als solcher erkannt und gemeldet wird. Ausgehend von den Gesundheitsbehörden würde dann eine Alarmierung der Sicherheitsbehörden erfolgen.

\section{Empfehlungen zum Vorgehen bei Pulverfunden}

\section{Einsatzleitung}

Besteht der Verdacht der erfolgten Ausbringung eines hochpathogenen Agens, obliegt den zuständigen Gesundheitsbehörden und in ausführender Tätigkeit der Amtsärztin oder dem Amtsarzt der Infektionsschutz, die Straf- und Tatverfolgung den örtlichen Polizeibehörden und Maßnahmen zur Personenrettung und Eindämmung der weiteren physikalischen Verbreitung der Kontamination der Feuerwehr, wobei der Gesundheitsschutz der 
Bevölkerung maßgeblich für das weitere Vorgehen ist [11]. Die Amtsärztin/der Amtsarzt ist immer hinzuzuziehen, wenn von einer biologischen Gefährdung ausgegangen wird. Ihr/ihm obliegt die Umsetzung der laut Infektionsschutzgesetz anzuwendenden Maßnahmen. Sie/er veranlasst auch eine mögliche Probenahme, das Einsenden der Proben an das Labor und empfängt die Befundmitteilung.

Da bei einem terroristischen Anschlag Folgeanschläge nicht auszuschließen sind, stellen die Tatortanalyse und Täterverfolgung durch die Polizeibehörden einen wesentlichen Beitrag zur Verhinderung weiterer Freisetzungen hochpathogener Agenzien und damit zum gesundheitlichen Bevölkerungsschutz dar. Maßnahmen zur Eindämmung der Verbreitung bzw. Beseitigung einer absichtlich ausgebrachten biologischen Kontamination sollten von der Einsatzleitung falls möglich so abgewogen und veranlasst werden, dass eine Spurensicherung durch die Polizei noch stattfinden kann.

\section{Probenahme und -versand}

Zunächst erfolgt nach Auffinden eines Pulvers die oben beschriebene Ernsthaftigkeitsprüfung. Wie erläutert, muss hierbei berücksichtigt werden, dass bei einem Pulverfund nicht automatisch eine Beimengung hochpathogener Agenzien angenommen werden sollte. Andere Materialien wie z. B. Sprengstoffe oder Industriechemikalien sind wesentlich leichter zugänglich. Bestätigt sich die Ernsthaftigkeit der Lage, wird dringend empfohlen, zunächst auf das Vorhandensein von konventionellen Stoffen (Sprengstoffe) zu überprüfen. Dieser Überprüfung folgt der analytische Ausschluss nicht konventioneller Stoffe, d. h. von radioaktiven Stoffen und flüchtigen chemischen Substanzen. Sind diese Überprüfungen negativ bzw. besteht weiterhin ein berechtigter Verdacht auf das Ausbringen hochpathogener Agenzien, so hat, sofern noch nicht geschehen, eine qualifizierte Probenahme der verdächtigen Substanz zu erfolgen. Primär ist danach der Versand in ein für die Analytik von Umweltproben auf hochpathogene Agenzien geeignetes Labor, nach den Regeln des Übereinkommens über die internationale Beförderung

Bundesgesundheitsbl 2015 · 58:699-704 DOI 10.1007/s00103-015-2160-3

(c) Springer-Verlag Berlin Heidelberg 2015

\section{Richter · C. Herzog}

\section{Allgemeines Vorgehen bei Verdacht auf einen Anschlag mit hochkontagiösen, hochpathogenen Agenzien}

\section{Zusammenfassung}

Der Verdacht auf das Ausbringen hochpathogener Agenzien, um der Bevölkerung bzw. Bevölkerungsgruppen oder Einzelpersonen Schaden zuzufügen, wird oftmals im Zusammenhang von Postsendungen ausgesprochen, die hauptsächlich, oder als Beistoff, undefinierbare Pulver mit oder ohne Drohschreiben enthalten. Bei anschließenden Untersuchungen wird von lokalen Einsatzkräften häufig der Ausschluss von Milzbranderregern oder deren Sporen angestrebt, was den Anschlägen in den USA aus dem Jahr 2001 mit milzbrandsporenhaltigem Pulver in Briefen zuzuschreiben ist. Bis zum jetzigen Zeitpunkt wurde jedoch in Deutschland bei „Pulversendungen" noch nie eine absichtliche Beimengung eines hochpathogenen Agens nachgewiesen. Zudem konnte in Deutschland bisher kein terroristischer Anschlag mit gefährlichen biologischen Agenzien weder in Form von Postsendungen noch anderen Ausbringungsmethoden verzeichnet werden. Auch kann im Anschlagsfall nicht davon ausgegangen werden, dass erneut Milzbrand- bakterien oder deren Sporen eingesetzt werden. Im Falle eines hinreichenden Verdachts auf einen Anschlag mittels Pulversendung reicht demnach weder die Überprüfung einer einzelnen Stoffgruppe (z.B. biologisch, chemisch, radioaktiv) noch der Ausschluss eines dezidierten Agens allein (z.B. Milzbranderreger) aus, um den Entscheidungsträgern entsprechende Informationen für das weitere Vorgehen an die Hand zu geben. Vielmehr sollte eine der jeweiligen Lage entsprechende Abfolge von Beratungs-, Ausschluss- und Messverfahren angestrebt werden, um systematisch alle relevanten Gefährdungen auszuschließen. Daher werden im Folgenden am Beispiel von Pulversendungen, Empfehlungen für das Vorgehen bei einem hinreichenden Verdacht auf das absichtliche Ausbringen gefährlicher biologischer Agenzien gegeben.

Schlüsselwörter

Pulverfunde · Lageeinschätzung · Schutzund Gegenmaßnahmen · Einsatzgruppe Bio . Probenahme und Forensik

\section{General procedures in response to suspected attacks with highly contagious and pathogenic agents}

\section{Abstract}

The discovery of undefined powders that are content of letters or parcels with or without threats in writing addressed to institutions, groups or persons, often raises the suspicion of an attack involving biological agents such as anthrax. Subsequent investigations and analyses by local authorities often aim at excluding anthrax or anthrax spores. Suspicion and actions are then mistakenly justified by referring to the 2001 anthrax letter attacks in the USA, which now lie more than 10 years in the past. In Germany to date there has never been a terrorist attack involving dangerous biological agents, neither in letters nor in any other possible form. In addition, it cannot be assumed that anthrax bacteria or spores will be used again in a bioterrorist event. In the case of a valid suspicion of a threatening letter, neither analyses to exclude only a certain group of substances (e.g. biological, chemical or radiological) nor analyses to exclude only one biological agent (e.g. anthrax) in particular would provide sufficient information for decision makers to conduct further actions in coping with the given situation. Moreover, a sequence of procedures such as consultation, exclusion, and analyses should be followed in order to systematically exclude all relevant threats. Therefore, and in respect to the current CBRNE threat assessment for Germany, the following article provides recommendations of actions to be taken for coping with a valid suspicion of an intentional release of biological agents utilizing powder letters as an example.

\section{Keywords}

Undefined powders - Situation reports .

Protective and counter measures - Bio incident response unit . Sampling and Forensics gefährlicher Güter auf der Straße (ADR) zu veranlassen. Dabei ist zu berücksichtigen, dass „bioterroristisches Verdachts- material“ grundsätzlich nach UN2814 klassifiziert ist und unter Beachtung der Verpackungsanweisung P620 zu versen- 


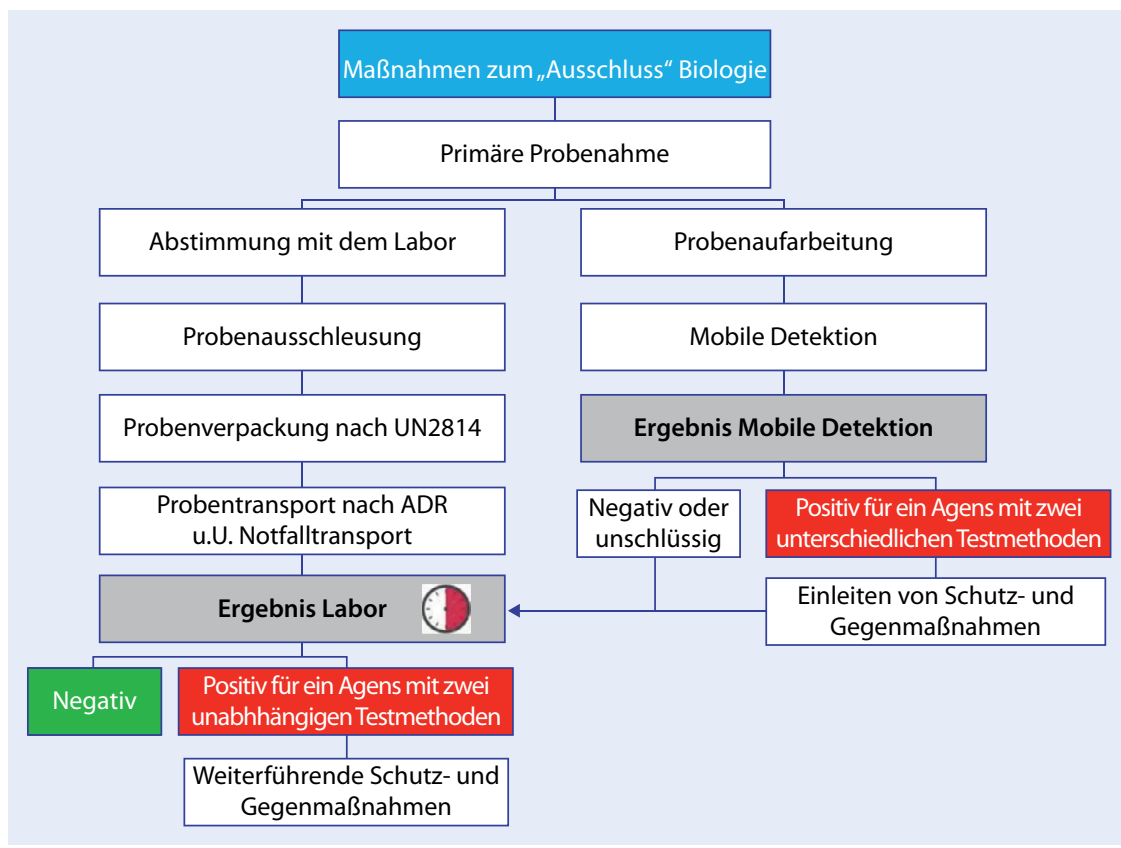

Abb. 3 A Vorgehen bei vermutlicher Ausbringung eines B-Agens; Maßnahmen zu Probennahme, Probentransport und Ausschluss von biologischen Stoffen unter Berücksichtigung der stationären (Labor) und mobilen Detektion (falls Verlässlichkeit und Reproduzierbarkeit der Methode gegeben ist). Das Laborergebnis sollte spätestens $6 \mathrm{~h}$ nach Probeneingang vorliegen. (ADR: Accord européen relatif au transport international des marchandises dangereuses par route = Übereinkommen über die internationale Beförderung gefährlicher Güter auf der Straße)

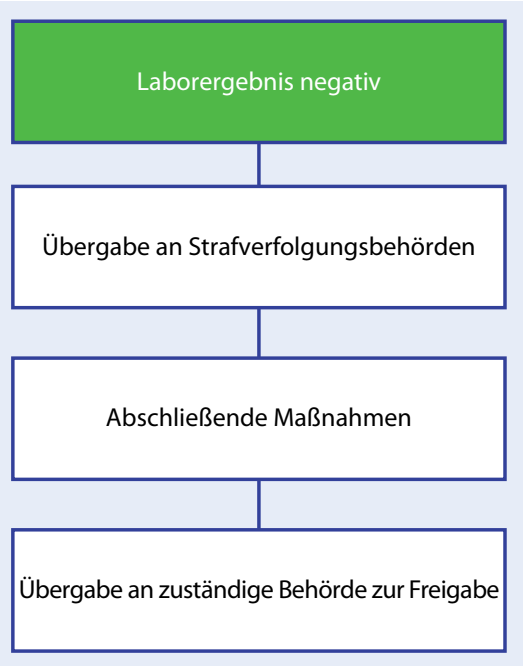

Abb. $4 \Delta$ Vorgehen bei vermutlicher Ausbringung eines B-Agens; weitere Schritte bei negativem Laborergebnis

den ist $[12,13]$. Die Probenahme erfolgt in der Regel durch spezialisierte Teams, zu deren Ausrüstung ohnehin die nach der ADR geforderten Verpackungsmaterialien gehören sollten. Eine Ausnahme dieser Vorschriften ist nur bei der akuten Gefahrenabwehr zur Rettung menschlichen Lebens gestattet, sollte aber durch spezia- lisierte Einheiten, die sich prinzipiell auf solche Lagen vorbereiten, nicht in Anspruch genommen werden müssen. In jedem Fall müssen aber der UN2814 und der P620 entsprechende Verfahren zum Kennzeichnen und Verpacken der Subs$\operatorname{tanz}$ eingehalten werden.

\section{Analyse der biologischen Probe}

In einigen Fällen kann erwogen werden, nebst der Analytik in einem stationären Labor, eine Schnelldiagnostik vor Ort durchzuführen. Jedoch existieren beim jetzigen Stand der Wissenschaft und Technik auf dem Gebiet der VorOrt-Schnelldiagnostik von hochpathogenen Agenzien berechtigte Zweifel an Anwendbarkeit und Interpretierbarkeit. Sowohl immunologisch-serologische Methoden, beispielsweise in Form von Lateral Flow Assays (LFAs), als auch Methoden auf der Basis der Polymerasekettenreaktion (PCR), die speziell für die Anwendung im Feld entwickelt wurden, eignen sich aufgrund ihrer Handlichkeit zwar prinzipiell für den Außeneinsatz, doch erzielen sie häufig unverlässliche, nicht reproduzierbare Ergebnisse (falsch positiv und falsch negativ Messungen, fehlerhafte Kontrollmessungen) [14-18]. Zudem korreliert die Verlässlichkeit der Methoden, die teilweise unter Laborbedingungen etabliert wurden, selten mit der unter Feldbedingungen, was die Aussagekraft solcher Schnelltests vermindert [14-16, 18]. Dennoch konnten in den letzten Jahren Fortschritte bei der Verbesserung dieser Parameter gemacht werden. Die Vorhaben zur Verbesserung von Systemen zur schnellen Vor-Ort-Analyse hochpathogener Agenzien sind in den letzten Jahren oft Bestandteil von national und international geförderten Verbundprojekten. Insofern ist bei jedem Einsatz individuell zu prüfen, ob ein Schnelltest zu einem verwertbaren Erkenntnisgewinn führt und darauf aufbauend Entscheidungen getroffen und Folgemaßnahmen veranlasst werden können. Zum jetzigen Zeitpunkt zählen der zügige Transport der Substanz und die Analyse in einem geeigneten stationären Labor als die wesentlichsten Maßnahmen zur Bestimmung des Agens.

Hierbei ist es wichtig, das entsprechende Labor im Vorfeld vor allem über das zu untersuchende Agenzienspektrum zu informieren, sodass es die zur Analyse notwendigen Vorbereitungen treffen kann. Im biologischen Bereich reicht es im Prinzip nie aus, „nur“ auf das Vorhandensein bzw. die Abwesenheit von einem Agens (beispielsweise Milzbranderregern) zu testen, sondern entsprechend dem Gefährdungspotenzial muss auch auf das Vorhandensein bzw. die Abwesenheit anderer hochkontagiöser bzw. hochpathogener Agenzien geprüft werden. Dabei korreliert das zu testende Agenzienspektrum mit der aktuellen Gefährdungsbewertung und unterliegt damit auch einer Dynamik. Es ist denkbar, dass abhängig vom zu untersuchenden Spektrum, auch mehrere Labore einbezogen werden. Es kann ggf. von Vorteil sein, den gesamten Verdachtsgegenstand zu verpacken und zur weiteren Analyse einem Labor zuzuführen. Limitierungen durch Größe und Gewicht sind individuell mit dem untersuchenden Labor abzustimmen. Nach erfolgter Analytik übermitteln dann die Labore die Ergebnisse und ggf. notwendigen Interpretationen ihrer Untersuchungen an den Einsender (Amtsärztin/Amtsarzt), die/der weitere Maßnahmen basie- 


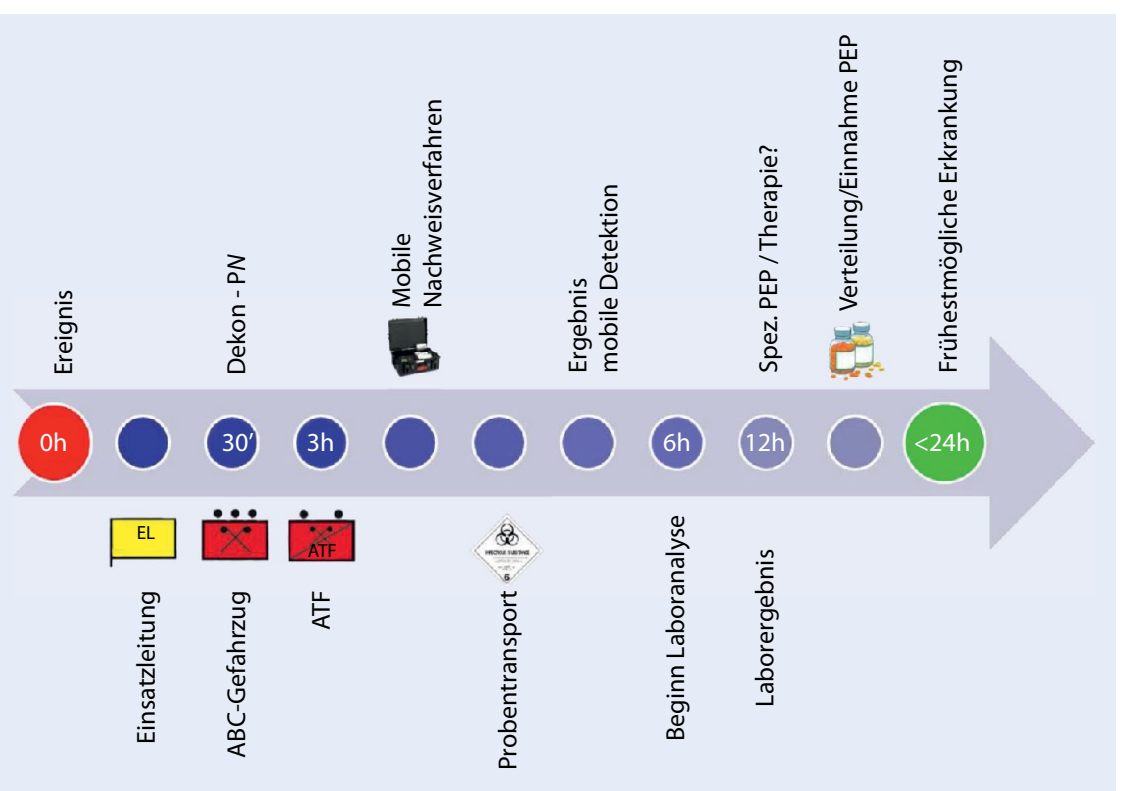

Abb. 5 ॥ Schematischer zeitlicher Ablauf von Maßnahmen aus Sicht der Probenahme, der Dekontamination, des Probentransportes, der Analytik und möglichen medizinischen Gegenmaßnahmen vom Anschlagsereignis bis Eintritt einer lebensbedrohlichen Erkrankung unter Berücksichtigung der bisher kürzesten beschriebenen Inkubationszeit von Infektionserregern. Um eine rechtzeitige Postexpositionsprophylaxe - sofern vorhanden - gewährleisten zu können, sollte die Probe im Idealfall spätestens $6 \mathrm{~h}$ nach Eintritt des Ereignisses im Labor sein und das Laborergebnis nach weiteren $6 \mathrm{~h}$ vorliegen. Zeitangaben zur groben Orientierung. (P, Personen; V, Verletzte; ATF, Analytische Task Force; PEP, Postexpositionsprophylaxe)

rend auf diesen Untersuchungsergebnissen veranlassen kann.

In den • Abb. 2-5 ist ein möglicher Ablauf der Maßnahmen schematisch dargestellt.

\section{Dekontamination}

Zur Probennahme muss eine Persönliche Schutzausrüstung (PSA) getragen werden. Bei jedem Einsatz von PSA ist eine standardmäßige Personendekontamination (Dekon P) sicherzustellen [19]. Dazu gehört u. a. die Einrichtung eines Dekonplatzes der Gefahrengruppe II und III, der spätestens 15 min nach Anlegen der PSA betriebsbereit sein muss [19]. Bei hinreichendem Verdacht auf eine absichtliche Ausbringung von hochpathogenen Agenzien ist nach Gefahrengruppe III zu verfahren. Das entsprechende Dekonmittel, bei dessen Auswahl das RKI ggf. unterstützen kann, muss zu dieser Zeit in ausreichenden Gebinden vorhanden und falls notwendig angemischt sein. Für Dekon-P-Maßnahmen sollten grundsätzlich spezielle „Dekoneinheiten“ alarmiert werden. Nur in begründeten Ausnahmefällen und nur, wenn es die Lage erfordert, kann zunächst mit einer Notdekon anstelle der kräfte, die zur Unterstützung bei akuten Lagen mit hinreichendem Verdacht auf das absichtliche Ausbringen von gefährlichen biologischen Agenzien herangezogen werden können und die ggf. umgehend handeln müssen, empfiehlt es sich, Möglichkeiten für eine Notdekontamination in der Ausrüstung für die eigenen Mitarbeiter mitzuführen, die aber nicht die standardmäßige Dekon P ersetzen soll.

\section{Unterstützung durch Spezialkräfte}

Beim Verdacht auf Freisetzung von hochpathogenen Agenzien hält der Bund Kapazitäten und Expertise zur Unterstützung und Beratung der vor Ort verantwortlichen Behörden vor. Ein solches Instrument stellt die Einsatzgruppe Bio am RKI dar [15]. Die Einsatzgruppe Bio bietet den für das Management einer außergewöhnlichen biologischen Lage verantwortlichen Behörden auf Anforderung Dekon P begonnen werden. Für Spezial- von der Beratung bis zur qualifizierten Umweltprobenahme vor Ort, dem Probentransport, der umfassenden Analytik und der Ergebnisinterpretation eine umfassende Unterstützung an. In Abstimmung mit dem ständigen Arbeitskreis der Kompetenz- und Behandlungszentren für hochkontagiöse und lebensbedrohliche Erkrankungen (STAKOB) hat die Einsatzgruppe Bio einen Zeitplan entwickelt, der den Zusammenhang zwischen Ereignis, Probenahme, Probentransport, Laborergebnis und ggf. möglicher Postexpositionsprophylaxe von betroffenen und potenziell mit einem hochpathogenen Agens infizierten Personen aufzeigt (- Abb. 5). Die Einsatzgruppe Bio ist damit ein Instrument, das bei einer solchen Lage auf konkrete Anforderung hin verantwortliche Institutionen unterstützt und berät.

Auch im Rahmen der bei Vorfällen mit gefährlichen Stoffen unterstützenden analytischen Task Force (ATF) in Deutschland wird momentan die Erweiterung auf eine Unterstützung zur Probennahme bei biologischen Gefahrenlagen unter Federführung des Bundesamtes für Bevölkerungsschutz und Katastrophenhilfe (BBK) evaluiert. Die endgültige Entscheidung darüber, ob und in welchem Umfang eine entsprechende Erweiterung stattfinden soll, wird im Anschluss an die momentan laufende Pilotphase getroffen. Dabei sind die ATF-Standorte zumeist einer Berufsfeuerwehr angegliedert und können dann ebenso auf die am RKI vorgehaltene Expertise zu hochpathogenen biologischen Agenzien zurückgreifen. Bisher wird im Bereich der ATF Unterstützung bei Probenahme und Analytik beim Verdacht auf Austritt und Verbreitung von chemischen oder radioaktiven Substanzen angeboten.

\section{Fazit}

Bis zum jetzigen Zeitpunkt wurde innerhalb Deutschlands noch nie eine Postsendung zugestellt, die tatsächlich hochpathogene Agenzien enthielt. Auch sonst kam es in Deutschland noch nicht zu einer in terroristischer Absicht durchgeführten Freisetzung hochpathogener Agenzien. Es wurde jedoch, besonders seit Bekanntwerden der Milzbrandbriefanschläge 2001 in den USA, eine Viel- 
zahl an Pulversendungen ohne absichtliche Beimengung hochpathogener Agenzien innerhalb Deutschlands an die verschiedensten Zielpersonen, Zielgruppen bzw. Institutionen versendet. Dieser Sachverhalt fließt in die momentanen Gefährdungsbewertungen ein und sollte somit auch bei der Ernsthaftigkeitsprüfung eventueller Lagen berücksichtigt werden. Dennoch bleibt es unerlässlich, Verfahrensabläufe zur Bewältigung einer realen Lage zu entwickeln und Spezialkräfte und Expertise für die Unterstützung von örtlichen Einsatzleitungen vorzuhalten. Auch wenn es momentan nur eine geringe Eintrittswahrscheinlichkeit für das absichtliche Verbreiten hochpathogener Agenzien gibt, so kann der tatsächliche Eintritt eines solchen Ereignisses zusätzlich zum Personenschaden auch weitere Auswirkungen auf die Gesellschaft haben. Daher bleibt das absichtliche Ausbringen von hochkontagiösen hochpathogenen Agenzien eine ernst zu nehmende abstrakte Gefahr.

\section{Korrespondenzadresse}

\section{Dr. M. Richter}

Zentrum für Biologische Gefahren und Spezielle Pathogene, Informationsstelle des Bundes für Biologische Gefahren und Spezielle Pathogene Robert Koch-Institut, Berlin RichterMar@rki.de

Danksagung. Das einsatztaktische Vorgehen bei der Freisetzung von gefährlichen biologischen Agenzien wurde gemeinsam mit dem Sachgebiet "Umwelt und Explosivstoffanalytik" des Landeskriminalamts Berlin entwickelt. Dafür und für die immer sachliche und konstruktive Zusammenarbeit sowie die Entwicklung weiterer gemeinsamer Vorgehensweisen mit dem Ziel der Gefahrenabwehr im gesundheitlichen Bereich bedankt sich der Autor. Frau Dr. Lee danke ich für die Mitarbeit an der Entwicklung von Kriterien zur Lagebeurteilung.

\section{Einhaltung ethischer Richtlinien}

Interessenkonflikt. M. Richter und C. Herzog geben an, dass kein Interessenkonflikt besteht.

Dieser Beitrag beinhaltet keine Studien an Menschen oder Tieren.

\section{Literatur}

1. Beeching NJ, Dance DaB, Miller ARO, Spencer RC (2002) Biological warfare and bioterrorism. BMJ 324:336-339

2. Jansen HJ, Breeveld FJ, Stijnis C, Grobusch MP (2014) Biological warfare, bioterrorism, and biocrime. Clin Microbiol Infect 20:488-496

3. Baize S, Pannetier D, Oestereich L et al (2014) Emergence of Zaire Ebola virus disease in Guinea. N Engl J Med 371:1418-1425

4. W.H.O. ERT (2014) Ebola virus disease in West Africa-the first 9 months of the epidemic and forward projections. N Engl J Med 371:1481-1495

5. Vogler AJ, Chan F, Nottingham R et al (2013) A decade of plague in Mahajanga, Madagascar: insights into the global maritime spread of pandemic plague. MBio 4:1-10

6. Fineberg HV (2014) Pandemic Preparedness and Response-Lessons from the H1N1 Influenza of 2009. N Engl J Med 370:1335-1342

7. Kr.Waff.Kontr.G. (1961) Gesetz über die Kontrolle von Kriegswaffen. In: KrWaffKontrG.

8. C.D.C. (2015) Bioterrorism Agents/Diseases. In: http://emergency.cdc.gov/agent/agentlist-category.asp. Centers for Disease Control and Prevention Atlanta, USA, p Bioterrorism Agents/Diseases

9. Pottage T, Goode E, Wyke S, Bennett AM (2014) Responding to biological incidents - what are the current issues in remediation of the contaminated environment? Environ Int 72:133-139

10. Dubber M, Hörnle T (2014) Criminal law: a comparative approach. Oxford University Press, United Kingdom

11. If.S.G. (2013) Infektionsschutzgesetz. In: IfSG.

12. Thurm V, Heinemann A (2010) Versand von medizinischem Untersuchungsmaterial: Sicher und vorschriftenkonform Literatur. Dtsch Arztebl 107:16

13. A.D.R. (2013) Europäisches Übereinkommen über die internationale Beförderung gefährlicher Güter auf der Straße. In: Union E (ed) European Union Brüssel

14. Sajid M, Kawde A-N, Daud M (2014) Designs, formats and applications of lateral flow assay: A literature review. J Saudi Chem Soc Article in press doi: 10.1016/j.jscs.2014.09.001

15. Richter M (2014) Operative Einsatzmöglichkeiten der "Informationsstelle des Bundes für Biologische Gefahren und spezielle Pathogene" (IBBS) am RKI. Gesundheitswesen 76:V27

16. Ngom B, Guo Y, Wang X, Bi D (2010) Development and application of lateral flow test strip technology for detection of infectious agents and chemical contaminants: a review. Anal Bioanal Chem 397:1113-1135

17. Li Z, Wang Y, Wang J, Tang Z, Pounds JG, Lin Y (2010) Rapid and sensitive detection of protein biomarker using a portable fluorescence biosensor based on quantum dots and a lateral flow test strip. Anal Chem 82:7008-7014

18. Yager P, Edwards T, Fu E et al (2006) Microfluidic diagnostic technologies for global public health. Nature 442:412-418

19. A.F. Kz.V. (2004) Feuerwehr-Dienstvorschrift 500. In: FwDV 500. Ausschuss Feuerwehrangelegenheiten, Katastrophenschutz und zivile Verteidigung (AFKzV), Germany 